\title{
INOVAÇÃO EM SOFTWARE AS A SERVICE: FUNCIONALIDADES EM FERRAMENTAS DE MONITORAMENTO DE MÍDIAS SOCIAIS
}

\author{
Innovation in Software as a Service: Functionalites in Social Media Monitoring \\ Tools
}

\begin{abstract}
Innovación en Software as a Service: funcionalidades en herramientes de monitoreo de medios sociales
\end{abstract}

Tarcízio Roberto da Silva

Universidade Metodista de São Paulo - UMESP

eu@tarciziosilva.com.br

\begin{abstract}
Resumo
O presente artigo busca descrever o lançamento de inovação incremental em softwares de monitoramento de mídias sociais. A tática, típica de produtos SaaS (software as a service) vai ao encontro do conceito de beta perpétuo, característico das mídias sociais e web 2.0. Como estudo de caso, trazemos a referência ao lançamento de módulos de análise de redes baseadas em grafos em 4 fornecedoras de serviço: Sysomos, Pulsar, Digimind e V-Tracker. Propõe-se a relevância da descrição e visibilidade destes atores organizacionais, sobretudo como atores condicionantes dos ambientes interacionais online a partir das relações entre inovação e particularidades da comunicação digital.
\end{abstract}

Palavras-chave: inovação, software as a service, mídias sociais, monitoramento de mídias sociais

\begin{abstract}
This article aims to describe the launch of incremental innovation in social media monitoring software. The tactic, typical of SaaS (software as a service) products, is in line with the concept of perpetual beta, which is characteristic of social media and web 2.0. As a case study, we bring the reference to the release of network-based analysis modules in four service providers: Sysomos, Pulsar, Digimind, V-Tracker. It is proposed the relevance of the description and visibility of these organizational actors, especially as conditioning actors of interactive online environments through the relation between innovation and particularities of digital communication.
\end{abstract}

Key words: innovation, software as a service, social media, social media monitoring

\section{Resumen}

El presente artículo busca describir el lanzamiento de innovación incremental en softwares de monitoreo de medios sociales. La táctica, típica de productos SaaS (software as a service) va al encuentro del concepto de beta perpetuo, característico de los medios sociales y web 2.0. Como estudio de caso, traemos la referencia al lanzamiento de módulos de análisis de redes basadas en grafos en 4 proveedores de servicio: Sysomos, Pulsar, Digimind, V-Tracker. Se 
propone la relevancia de la descripción y visibilidad de estos actores organizacionales, sobre todo como actores condicionantes de los ambientes interactivos online a partir de las relaciones entre innovación y particularidades de la comunicación digital.

Palabras clave: Innovación, software as a service, medios sociales, monitoreo de medios sociales

\section{MÍDIAS SOCIAIS, “REDES SOCIAIS” E NOVOS MERCADOS}

Ao longo dos últimos 10 anos, plataformas de mídias sociais como Orkut, MySpace, Facebook e Twitter ganharam destaque como negócios inovadores, gerando amplo debate sobre seus impactos na economia, política, mídia, comunicação e até aspectos psicossociais. Hoje, entre os sites mais visitados do mundo ocidental temos o Facebook com 1,59 bilhões de usuários, Tumblr com 555 milhões, Instagram com 400 milhões e Twitter com mais de 320 milhões de usuários ${ }^{1}$.

Entre os conceitos definidores mais referenciados sobre "mídias sociais", vale mencionar o trabalho de Haenlein e Kaplan que propôs que "Social Media is a group of Internet-based applications that build on the ideological and technological foundations of Web 2.0, and that allow the creation and exchange of User Generated Content." (2010, p.61). O termo "conteúdo gerado pelo usuário" teve seu boom de interesse entre 2006 e $2008^{2}$, período no qual os serviços comerciais de comunicação (sobretudo nas áreas de publicidade e relações públicas) se estruturaram ao redor do mundo em agências e empresas que buscavam entender este novo panorama e lucrar através de sua operacionalização para todos os tipos de negócios. A histórica capa da Time que colocou "Você" ("You") como a "Pessoa do Ano" ("Person of the Year") deu o reconhecimento definitivo às mídias sociais como Facebook, Twitter e YouTube que ganhavam popularidade meteórica ${ }^{3}$. Mas, para além dos ganhos de valor em Bolsas de Valores e sistemas de publicidade, estes sites começaram a ser vistos como locais de intervenção criativa por profissionais de publicidade, marketing, relações públicas e áreas afins. Em documento publicado pelo Interactive Advertising Bureau no início de 2008, a seguinte definição pode ser lida:

UGC has been around in one form or another since the earliest days of the Internet itself. But in the past five years, thanks to the growing availability of

\footnotetext{
${ }^{1}$ Dados do portal Statista http://www.statista.com/statistics/272014/global-social-networks-ranked-by-numberof-users/

${ }^{2}$ Como dados obtidos do Google Trends atestam -

https://www.google.com.br/trends/explore\#q=user\%20generated $\% 20$ content

${ }^{3} \mathrm{http}: / /$ content.time.com/time/specials/packages/0,28757,2019341,00.html
} 
high-speed Internet access and search technology, it has become one of the dominant forms of global media. It is currently one of the fastest growing forms of content on the Internet. UGC is fundamentally altering how audiences interact with the Internet, and how advertisers reach those audiences. (IAB, 2008, p.2)

Como representante da indústria da "publicidade interativa", a IAB tenta consolidar neste documento algumas boas práticas para agências e profissionais do segmento enfrentarem o novo panorama estabelecido a partir da liberação do pólo de emissão na cibercultura (LEMOS, 2003). Nas palavras do autor,

o que está em jogo como o excesso de informação nada mais é do que a emergência de vozes e discursos anteriormente reprimidos pela edição da informação pelos mass media. A liberação do pólo da emissão está presente nas novas formas de relacionamento social, de disponibilização da informação e na opinião e movimentação social da rede. Assim chats, weblogs, sites, listas, novas modalidades midiáticas, e-mails, comunidades virtuais, entre outras formas sociais podem ser compreendidas por essa segunda lei [da Cibercultura]" (LEMOS, 2003, p. 20).

Neste efervescente período também são lançadas empresas que passam a definir os padrões para o trabalho em monitoramento de mídias sociais ou social listening. A Radian6, que anos depois faz história ao ser adquirida por 326 milhões de dólares ${ }^{4}$, é lançada em 2006. Neste e no ano seguinte, algumas das líderes atuais do mercado (como veremos ao tratar do Forrester Research Social Listening Report a seguir): Synthesio, Brandwatch e Sysomos são algumas delas. No Brasil, o mercado de monitoramento de mídias sociais passa a se estruturar entre 2009 e 2010, com o lançamento da atual líder Scup e concorrentes relevantes como Seekr e Livebuzz.

Mas o que compõe as mídias sociais e, mais especificamente, os sites de redes sociais? Que elementos os diferenciam de outros ambientes da internet? Também em 2008 uma edição do Journal of Computer-Mediated Communication, Boyd e Ellison definem os sites de redes sociais como

serviços de web que permitem aos usuários (1) construir um perfil público ou semipúblico dentro de um sistema conectado, (2) articular uma lista de outros usuários com os quais eles compartilham uma conexão e (3) ver e mover-se pela sua lista de conexões e pela dos outros usuários (BOYD e ELLISON, 2008. p.211).

Desta definição podemos depreender a relevância das conexões - não por acaso o termo "rede social" se tornou sinônimo popular destes sites. Além do papel da existência de

\footnotetext{
${ }^{4}$ https://www.crunchbase.com/organization/radian6\#/entity
} 
um perfil público ou semipúblico que representa indivíduos em suas presenças digitais acessíveis 24 horas por dia, os outros dois itens da definição tratam da descoberta e navegação de outros através das conexões. Entretanto, ao longo do tempo, o fluxo de conteúdo nestes sites ganha mais e mais relevância, como o lançamento dos recursos de "Timeline"5, para enfatizar a temporalidade do conteúdo relacionado a pessoas, organizações e eventos não só no presente, mas quanto ao passado e futuro também.

As autoras revisitam o conceito, respostas e críticas ao trabalho nos anos seguintes ao publicar em 2013 uma nova proposição da definição:

web-based services that allow individuals to (1) construct a public or semipublic profile within a bounded system, (2) articulate a list of other users with whom they share a connection, and (3) view and traverse their list of connections and those made by others within the system. The nature and nomenclature of these connections may vary from site to site. (ELLISON \& BOYD, 2013).

O primeiro traço característico sofre uma modificação relevante ao incluir que os perfis também consistem de conteúdo adicionado não só pelos usuários, mas também pelo sistema e por outros. Esta característica é central para o negócio do mercado de monitoramento de mídias sociais, uma vez que dinâmicas como persistência, replicabilidade, escalabilidade e buscabilidade (BOYD, 2011, p.46) transformam as conversações online e marketing boca a boca (KIETZMANN \& CANHOTO, 2013) sobre marcas e indivíduos componentes de suas imagens.

O terceiro traço, na nova definição, enfatiza a ideia de "fluxo": os conteúdos são publicados continuamente sobre múltiplos interesses, traços, características e atividades dos usuários. Assim a ideia de que o monitoramento de mídias sociais seria um método de pesquisa com a vantagem da espontaneidade do cotidiano ganha força, pois os conteúdos publicados pelos usuários de forma natural "depicts their innermost feelings and daily activities, which bestows upon analysts a unique opportunity to gain insight from people of the kind that has traditionally been available through polling and surveys." (AMPOFO, 2011, p.27) 


\section{FERRAMENTA DE MONITORAMENTO DE MÍDIAS SOCIAIS: DEFINIÇÃO E STATUS}

O novo panorama da comunicação "opens up great potential for the early recognition of issues, topics and trends, of corporate risks, market potential and opportunities for innovation” (GRUBLBAUER \& HARIC, 2013, p.686). Entre aplicações para marketing, publicidade e pesquisa, as práticas de monitoramento de mídias sociais podem ser descritas:

coleta, armazenamento, classificação, categorização, adição de informações e análise de menções online públicas a determinado(s) termo(s) previamente definido(s) e seus emissores, com os objetivos de: (a) identificar e analisar reações, sentimentos e desejos relativos a produtos, entidades e campanhas; (b) conhecer melhor os públicos pertinentes; e (c) realizar ações reativas e pró-ativas para alcançar os objetivos da organização ou pessoa de forma ética e sustentável. (SILVA, 2010, p.43)

Ampofo apresenta definição focada nos aspectos da publicação ao dizer que monitoramento de mídias sociais em tempo real "is the aggregation and analysis of social media content, as it is published, from social media portals" (2011, p.23). As aplicações do monitoramento são múltiplas: gerenciamento de reputação; detecção de eventos e gerenciamento de crises; análise de competição; monitoramento de campanhas; descoberta de tendências de mercado; pesquisa de mercado; detecção de influenciadores; customerrelationship management; e inovação em produtos (STAVRAKANTONAKIS, 2012, p.54).

Existem mais de 500 softwares do tipo (GILLIAT, 2016) e as líderes no mercado brasileiro são empresas locais lideradas por Scup (comprada pela internacional Sprinklr em 2015) e Seekr (SIRI, 2015). Estima-se o mercado de social business software - que inclui ferramentas de monitoramento de mídias sociais e automação - movimentou USD 13,9 bilhões em 2014 que poderá chegar a USD 37 bilhões em $2019^{6}$.

Técnica, a definição de ferramenta de monitoramento de mídias sociais de Laine e Fruhwirth:

software services offered over the Internet to filter and analyze the textual content produced by and in social media. The tools find content based on user-defined keywords. The tools incorporate multiple functionalities, such as analyse of volume, source, author, keyword, region and sentiment, and then reporting these analyses conveniently and in a graphical fashion. Most often the tools are based upon reading and aggregating multiple freely accessible RSS-feeds of updates from the social media websites (LAINE \& FRUHWIRTH, 2010, p.195)

\footnotetext{
${ }^{6} \mathrm{http}: / /$ marketingland.com/social-business-software-market-expected-to-reach-37-billion-by-2019-128217
} 
Tornou-se datada a concepção de que os dados são "gratuitamente acessíveis", uma vez que intermediários entre as mídias sociais e as ferramentas de monitoramento, como Gnip e Datasift (BATRINCA \& TRELEAVEN, 2015) ganharam mercado e se tornaram essenciais para as ferramentas enterprise. Porém, as principais funcionalidades apresentadas pelos autores permanecem como centrais nestes softwares.

Um expediente útil para documentar estas funcionalidades é recorrer aos rankings de produtos desenvolvidos por consultorias de pesquisa e auditoria. Como explicam Pollock e D'Adderio, "there is growing evidence to suggest that rankings play an enhanced role in decision-making" (POLLOCK \& D'ADDERIO, 2012, p.2). Na Figura 1, podemos ver o "The Forrester Wave", gráfico característico de relatórios sobre softwares produzidos pela Forrester Research. Publicado no primeiro trimestre de 2016, o relatório sobre softwares de “enterprise social listening” possui circulação limitada, pois é oferecido comercialmente, mas sumários executivos são divulgados pelas empresas mapeadas ${ }^{7}$. Exibe 12 ferramentas avaliadas em variáveis de Presença de Mercado, Oferta Atual (Current Offering) e Estratégia. As variáveis analisadas sobre aspectos técnicos da Oferta Atual são Informação; Fontes de Dados; Processamento de Dados; Funcionalidades de Dashboard; Interação. Serviços de Consultoria e Análise.

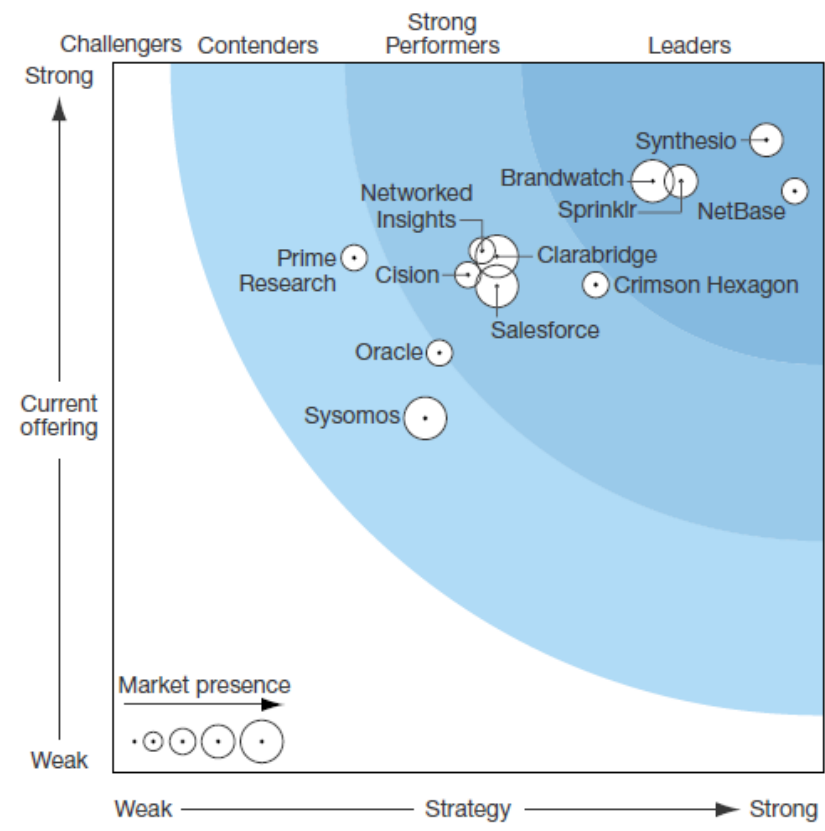

Figura 1: The Forrester Wave ${ }^{\mathrm{TM}}$ : Enterprise Social Listening Q1 2016

${ }^{7}$ Ver https://www.forrester.com/report/The+Forrester+Wave+Enterprise+Social+Listening+Platforms+Q1+2016/-/ERES122523 e https://www.sprinklr.com/pt-br/the-way/forrester-listening-wave-2016/ 
Com modelo similar, mas baseada em avaliações peer-to-peer, a G2 Crowd procura representar e ranquear os softwares de monitoramento de mídias sociais. Para qualificarem na categoria, os softwares devem englobar as possibilidades: "Listen for specific mentions across social media; Identify trending topics or phrases; Detail customer sentiment; Organize customer information; Identify thought leaders and influencers" ${ }^{\prime}$.

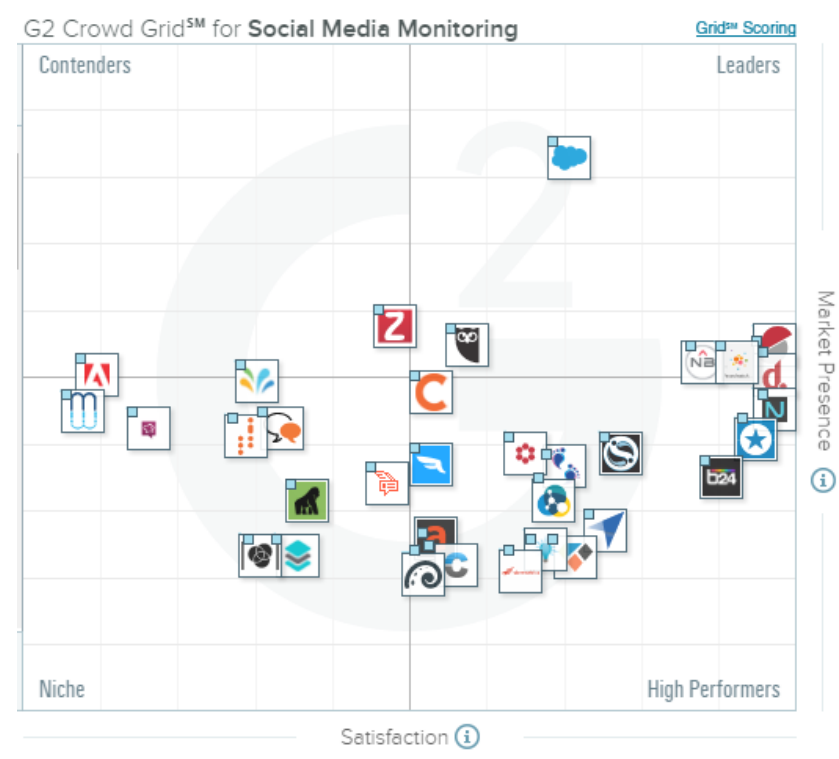

Figura 2: G2 Crowd Grid for Social Media Monitoring

Por fim, como último exemplo, a Goldbach Interactive, consultoria digital suíça, publica o documento bilíngue (alemão e inglês) Die Besten Social Media Monitoring Tools 2015 . Entre os itens analisados estão: Capacidades de Busca; Cobertura de Fontes; Análise de Mídia Espontânea; Engajamento e Mídia Própria; Funcionalidades de CRM; Alertas e Relatórios; Performance e Volume de Dados; Usabilidade. A partir da análise de 40 fornecedores de soluções de monitoramento, a empresa oferece análise detalhada em formato de texto e infográfico, incluindo ranking, notas para os diferentes critérios e identificação de tendências.

\footnotetext{
${ }^{8} \mathrm{https} / / / \mathrm{www} . \mathrm{g} 2 \mathrm{crowd} . \mathrm{com} /$ categories/social-media-monitoring

${ }^{9}$ https://www.goldbachinteractive.ch/insights/fachartikel/tool-report-2015
} 


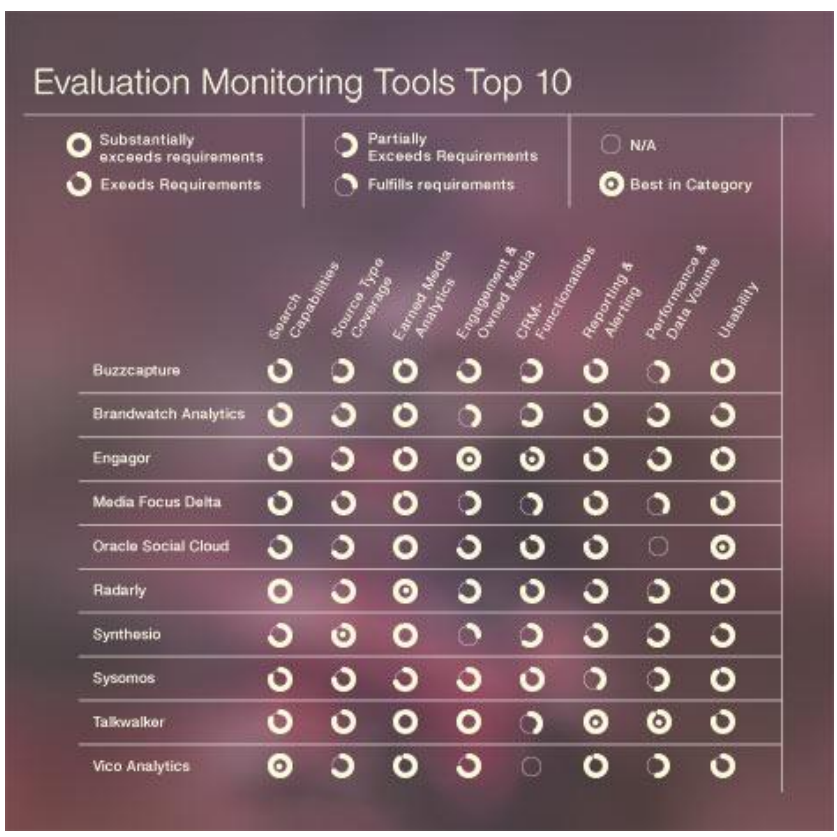

Figura 3: Goldbach Interactive - Evaluation Monitoring Tools Top 10

Rankings não são mecanismos nem puramente objetivos nem independentes do mercado que descrevem. Possuem impactos constitutivos ao selecionar determinadas características e itens a serem avaliados. Representam uma concepção do que merece atenção e "do more than simply grade or describe: they also offer new interpretations of a situation. Actors then adapt their behaviour to conform with this altered understanding" (POLLOCK \& D'ADDERIO, 2012, p.3).

Podemos, dessa forma, tomar como fato que existem alguns consensus quanto a funcionalidades padronizadas oferecidas pelas ferramentas de monitoramento de mídias sociais, que constituem e são constituídas por rankings como os citados. Para alcançar os objetivos deste artigo, vamos discorrer sobre três softwares que apresentaram entre 2014 e 2015 como inovação incremental a visualização de redes e grafos: a ferramenta canadense Sysomos, as americanas Pulsar e Digimind e a brasileira V-Tracker. Antes de tratar destas de forma específica, é necessário contextualizar este Mercado no panorama do modelo de negócio software as a service, assim como falar da visualização de redes e grafos enquanto metodologia inspirada em metodologias científicas e em outros softwares. 


\title{
3 SOFTWARE AS A SERVICE E VISUALIZAÇÃO DE REDES E GRAFOS: TRÊS CASOS DE INOVAÇÃO INCREMENTAL
}

Para além da definição do relevante mercado de monitoramento de mídias sociais em suas particularidades, devemos posicionar as práticas e serviços no panorama de seus modelos de negócio. São programas oferecidos no modelo Software as a Service (SaaS), definidos pela consultoria Gartner como:

\begin{abstract}
Software that"s owned delivered and managed remotely by one or more providers. [...] The provider delivers an application based on a single set of common code and data definitions, which are consumed in a one-to-many model by all contracted customers anytime on a pay-for-use basis, or as a subscription based on use metrics (Clark et al 2006 apud CHUKAROVA \& MIKHRAMOVA, 2010, p.6).
\end{abstract}

Esta lógica de software como serviço acessível a partir da internet traz em seu núcleo a ideia de inovação contínua como chave para manutenção dos negócios em ambientes cada vez mais competitivos. Traz um paralelo com a noção citada de "beta perpétuo" (O’REILLY, 2005) que impacta também a ponta final do processo: os cidadãos, consumidores ou usuários, a depender do ponto de vista, devem ser continuamente surpreendidos com novos recursos e desenhos da comunicação nos ambientes chamados de "sociais" na internet. Assim, o ciclo de inovação das abordagens neo-schumpeterianas (invenção, inovação, imitação ou difusão) é propulsionado pela evolução cada vez mais rápidas em aspectos técnicos que materializam a inovação aberta (SILVA, JUNQUEIRA \& CARDOSO, 2016, p. 109).

Os impactos das tecnologias digitais e amplitude da era informacional são vistos por pensadores da inovação como condicionantes do atual paradigma do capitalismo informacional e seus desdobramentos nas firmas e indústrias de áreas tão abrangentes como comércio e indústria. A particularidade aqui, ao tratarmos de cibercultura e seus negócios digitais como plataformas de mídias sociais, é que os novos recursos são desenvolvidos em uma contínua reapropriação das tensões entre abertura e concentração dos meios.

Os fornecedores de monitoramento de mídias sociais são utilizados por centenas de clientes. Com vasta amplitude de planos comerciais, de modelos freemium e mensalidades de centenas de reais até planos customizados que custam dezenas de mil reais, se baseiam sobretudo em métricas pay-for-use como número de mensagens ou ocorrências coletadas (como tweets, postagens no Facebook e blogs, vídeos YouTube ou notícias). A grande maioria oferece o modelo trial de acesso, período de teste gratuito com capacidade de coleta 
limitada e geralmente através de cadastro automatizado nos websites. A relativa facilidade de uso das ferramentas e alta competitividade do segmento podem explicar a característica.

As imagens abaixo dão uma boa ideia da similaridade de recursos das ferramentas. São respectivamente excertos retirados do Scup, Seekr e Vtracker. As imagens mostram primeiro a página de visualização de análise das ocorrências coletadas. Além da exibição do conteúdo (avatar do usuário, links e conteúdo da mensagem), é possível ver também botões e áreas para seleção de Sentimento (Positivo/Negativo/Neutro) e inserção de tags (códigos de análise que permitem quantificar variáveis observadas pelos analistas).

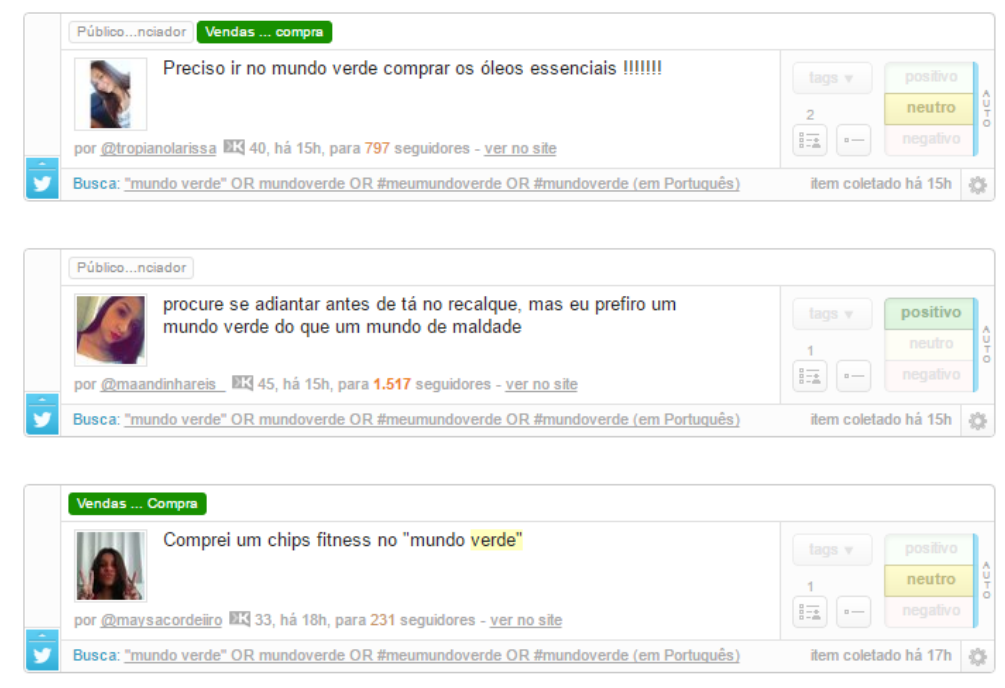

Figura 4: Tela de ocorrências no Scup

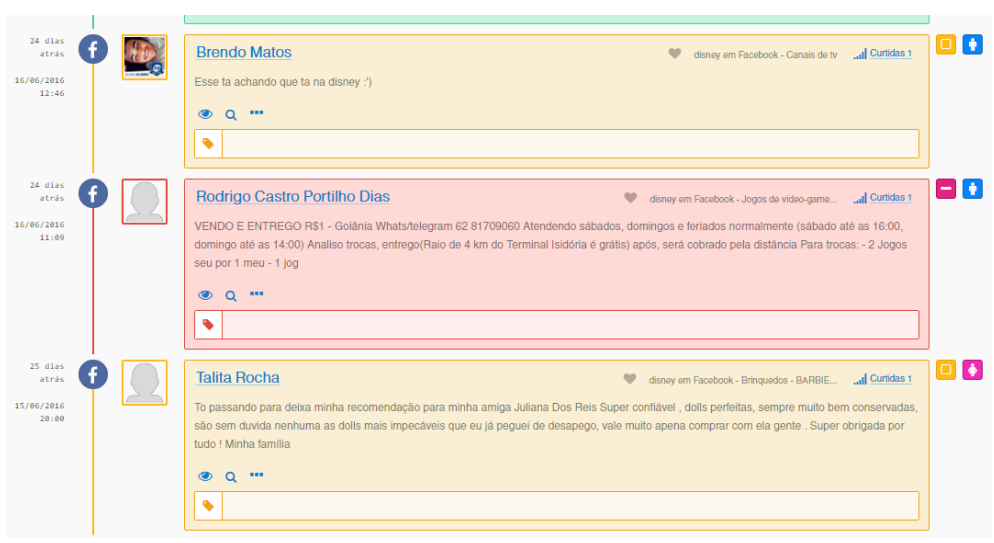

Figura 5: Tela de ocorrências no Seekr 


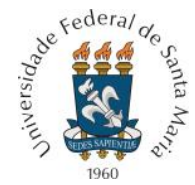

PROGRAMA DE PÓS-GRADUAÇÃO EM COMUNICAÇÃO DA UNIVERSIDADE FEDERAL DE SANTA MARIA

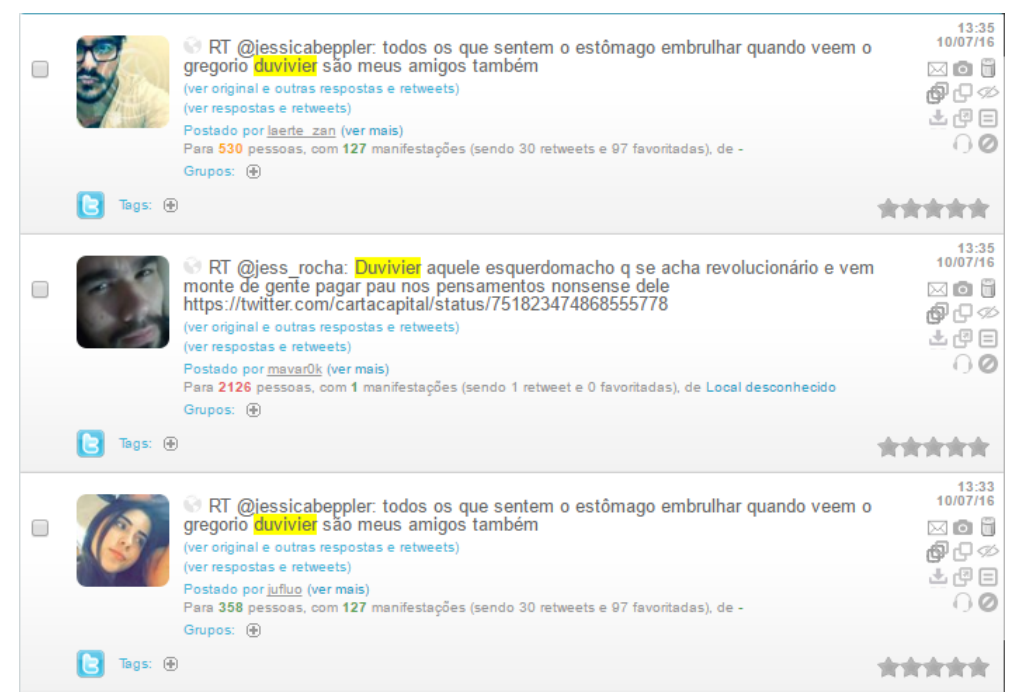

Figura 6: Tela de ocorrências na vTracker

Abaixo, telas de Relatórios, onde gráficos exibem dados de Distribuição de Sentimento, Volume de Menções e Principais Tags (códigos de análise).

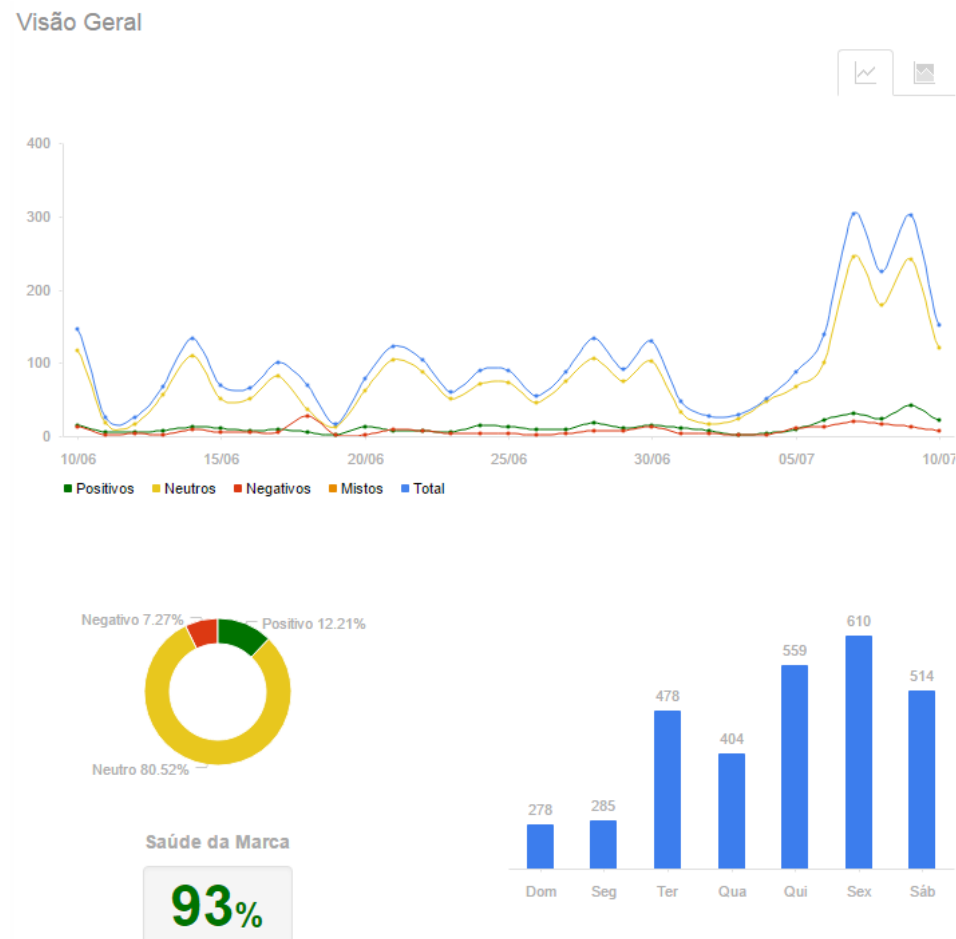

Figura 7: Tela da página de relatórios do Scup 


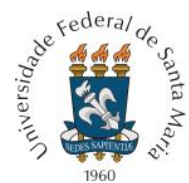

PROGRAMA DE PÓS-GRADUAÇÃO EM COMUNICAÇÃO DA UNIVERSIDADE FEDERAL DE SANTA MARIA

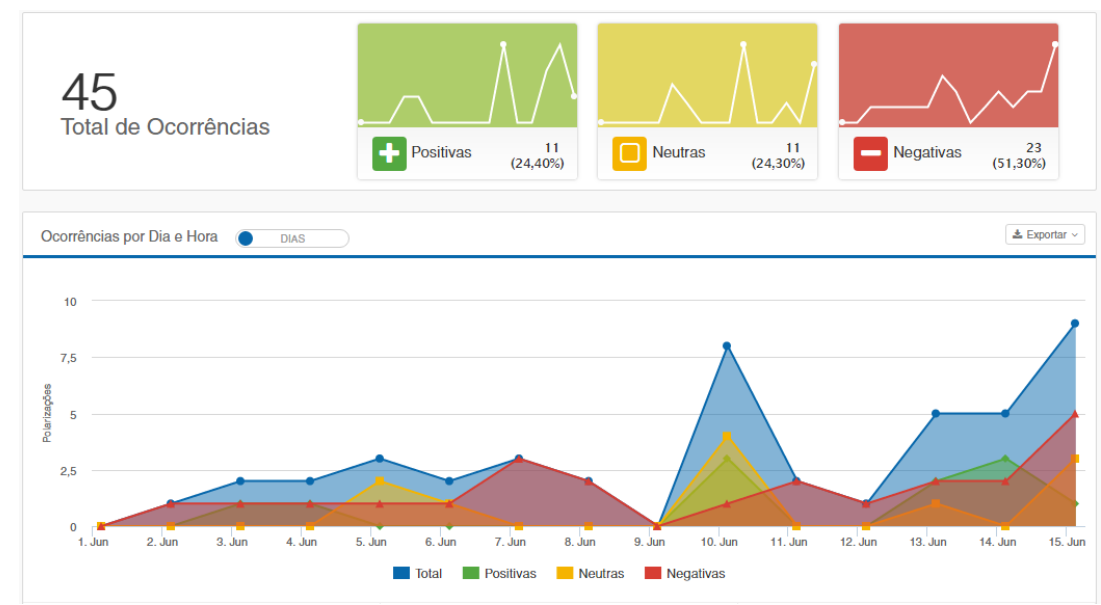

Figura 8: Tela da página de relatórios do Seekr

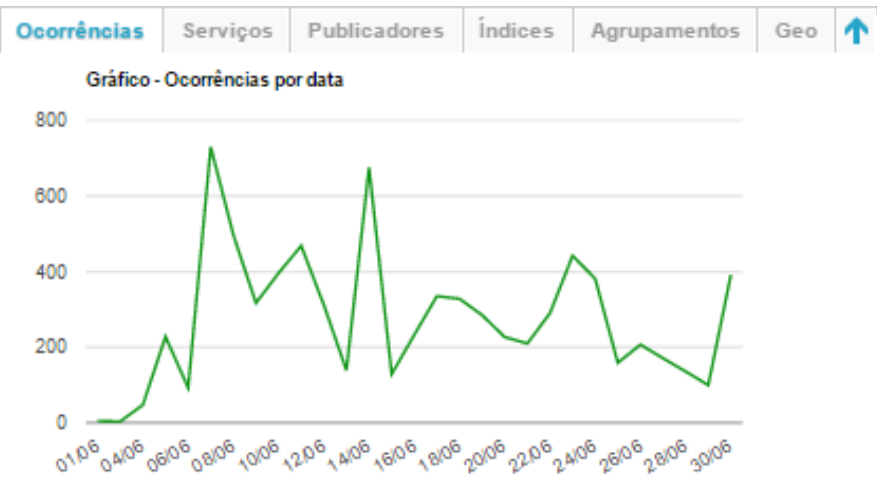

Gráfico - Ocorrências por sexo

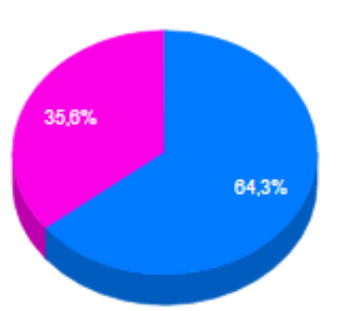

Gráfico - Ocorrências por sexo por qualificação

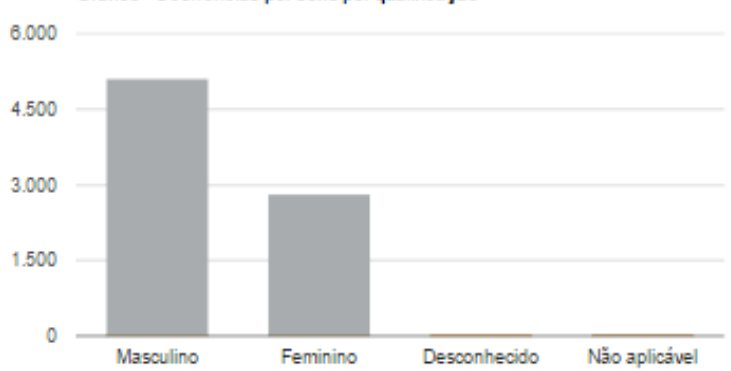

Figura 9: Tela de página de relatórios da V-Tracker 
Esta similaridade cria desafios de posicionamento dos fornecedores de monitoramento de mídias sociais, que tentam competir em termos de posicionamento no mercado. Troca de software traz problemas de gestão, então "industry may be still satisfied with the current solution, and consequently will be reluctant to change, even if the new solution is superior in terms of technology or service delivery." (CHUKAROVA \& MIKHRAMOVA, 2010, p.70).

Inovações e melhorias contínuas, portanto, se fazem necessárias para gerar interesse de novos clientes, sendo o caso da análise e visualização de redes e grafos. O termo "análise de redes sociais" ou "social network analysis" no inglês não é novo, uma vez que dá conta de metodologia de pesquisa proveniente de interfaces entre as ciências sociais e matemática já há algumas décadas. Tem sido utilizado como sinônimo de análise de conteúdo publicado nas redes sociais online, mesmo quando não veem os conteúdos e atores em estrutura de rede.

A interconectividade dos indivíduos em um mundo globalizado é comumente referenciada em termos de "grau de separação". Cada pessoa estaria a poucos "passos" de qualquer outra no mundo, medida empiricamente através de experimentos na década de 1970 (MILGRAM, 1972) e pelo próprio Facebook. Cientistas da plataforma em diversos momentos (BACKSTROM et al, 2012; EDUNOV et al, 2016) demonstraram a diminuição dos graus de separação nas redes do site ao longo dos anos. Como explica Recuero:

é o surgimento dessa possibilidade de estudo das interações e conversações através dos rastros deixados na Internet que dá novo fôlego à perspectiva de estudo de redes sociais a partir do início da década de 90. É, neste âmbito, que a rede como metáfora estrutural para a compreensão dos grupos expressos na Internet é utilizada através da perspectiva de rede social (RECUERO, 2009, p.24).

A análise estrutural de redes sociais se baseia em três pilares: os nós, vértices ou pontos, que sempre representam, no caso de redes sociais, pessoas ou organizações; as conexões, arestas ou linhas, que representam as relações entre estes nós; e as métricas de redes, que permitem identificar, através de parâmetros definidos, o tamanho da rede, centralidade dos nós, graus de entrada e saída etc.

O Gephi é um dos principais softwares para visualização de redes utilizados hoje no mundo por pesquisadores. Como explicam alguns de seus criadores,

In the aim of understanding networks, the visualization of large graphs has been developed for many years in many successful projects. Visualizations are useful to leverage the perceptual abilities of humans to find features in network structure and data. (BASTIAN, HEYMANN \& JACOMY, 2009, p.361) 


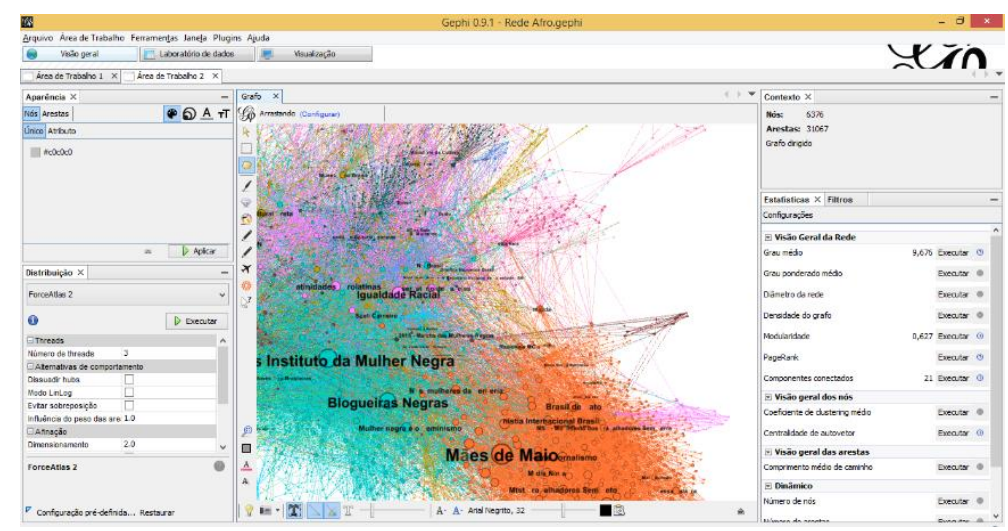

Figura 10: Tela do software Gephi

Pesquisadores interessados em estudar as mídias sociais elegem sobretudo o Gephi para a análise de dados, inclusive no Brasil (REGATTIERI et al, 2014; RECUERO, ZAGO \& BASTOS, 2014). A análise de estruturas sociais online pode

"provide insights into the interleaving of human interactions with the technological platforms used to enable and support them: the specific affordances of different social networking sites are also reflected in the structure of the social networks which form around them, if not always in the ways intended by their designers." (BRUNS, 2012, 1328).

A relevância da compreensão da sociedade através das redes torna-se um consenso ao ponto de existirem iniciativas de promoção da educação sobre redes, como o Network Science in Education ${ }^{10}$, que busca engajar jovens e adultos a estudar ciência de redes. Para a organização, "visualization of a network often helps to understand it and communicate ideas about connectivity in an intuitive, non-technical way" (NETSCIED, 2015, p.6).

Não é por acaso, então, que a Sysomos, empresa mais avançada e melhor posicionada no mercado do que as concorrentes citadas, enfatiza a simplicidade ao apresentar sua visualização de grafos. A descoberta de influenciadores é principal apelo comercial utilizado, como podemos ler na ocasião de lançamento: "Sysomos Heartbeat's new Influencer Communities makes it super simple for you to identify, measure and track people who hold the key to your social media success.",11

\footnotetext{
${ }^{10} \mathrm{http}: / /$ nysci.org/projects-main/netscied/

${ }^{11}$ https://blog.sysomos.com/2014/02/06/updates-to-map-heartbeat-powered-by-sysomos-influencer-communitiesinstagram-and-more/
} 


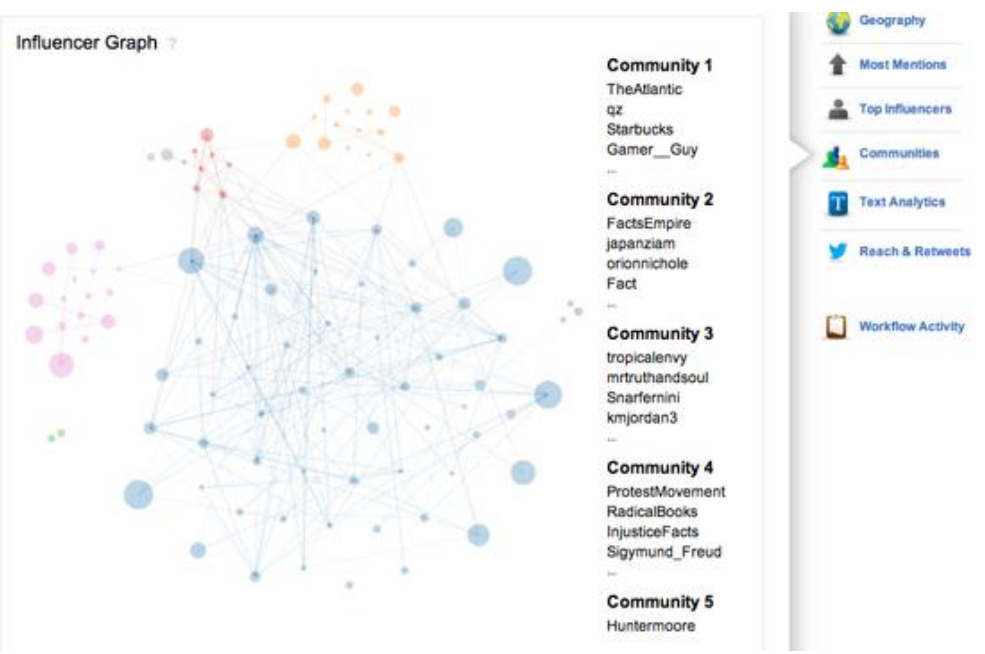

Figura 11: "Influencer Graph' da Sysomos

Em documento com estudo de caso mais detalhado ${ }^{12}$, explica o papel do Gephi no desenvolvimento da solução: "The implementation of the community detection algorithm uses Gephi, an open source graph analysis package, and the visualization is done using both Gephi and the D3 javascript library".

Trata-se de uma reapropriação de software de código aberto, fenômeno notável em inovações de produto, que podem "utilizar novos conhecimentos ou tecnologias, ou podem basear-se em novos usos ou combinações para conhecimentos ou tecnologias existentes" (OECD, 1997, p.57). Na descrição da funcionalidade oferecida pelo software Vtracker, esta combinação fica explícita, mas com uma hierarquização ${ }^{13}$. Apresenta-se como alternativa ao software Gephi, com supostas vantagens de menor curva de aprendizado e capacidade computacional:

"E como você pode ter isso? Baixando o Gephi, lendo 300 tutoriais e depois fritando o seu computador processando o grafo (para quem nunca tentou, essa operação utiliza muito poder computacional, esquentando muito, literalmente, o seu computador!)?"

\footnotetext{
12 http://www.marketwired.com/solutions/pdf/influencer-communities

${ }^{13}$ http://www.vtracker.com.br/blog/2014/08/nova-funcionalidade-analise-e-geracao-automatica-de-grafos/
} 


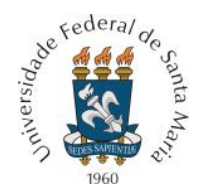

PROGRAMA DE PÓS-GRADUAÇÃO EM COMUNICAÇÃO DA UNIVERSIDADE FEDERAL DE SANTA MARIA

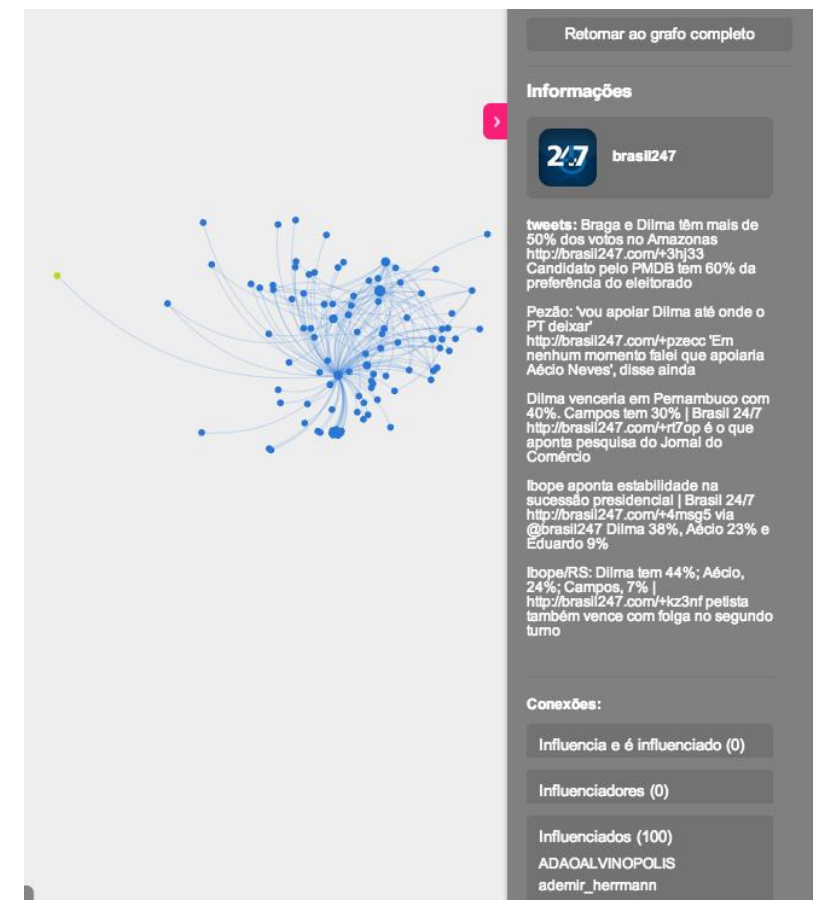

Figura 12: Tela de funcionalidade de grafos da V-Tracker

A Pulsar Analytics, por sua vez, não diminui a importância do Gephi, deixando explícitas duas fases no desenvolvimento da solução ${ }^{14}$ :

"Last year we released the first integration with Gephi that gives our users the ability to plug their social data directly into Gephi. This year we released a new interactive graph visualisation that shows the core of any network right in the browser"

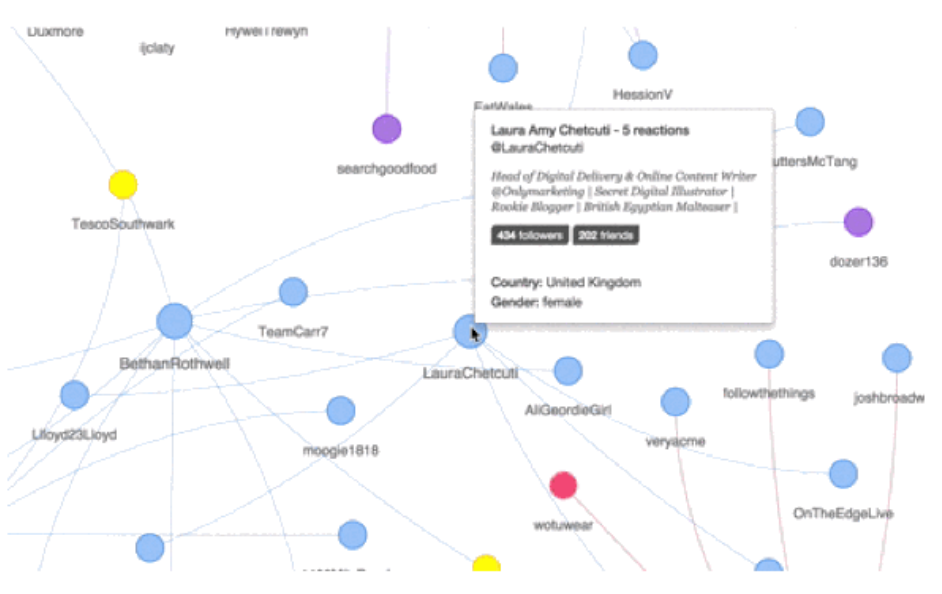

\footnotetext{
${ }^{14} \mathrm{http}: / /$ www.pulsarplatform.com/blog/2015/pulsar-top-10-innovations-of-2015-how-were-changing-the-gamein-the-social-listening-industry/
} 
A Digimind ${ }^{15}$, por fim, traz a visualização mais simples (sem algoritmos de clustering e layout force-directed), e realiza a divulgação principal da funcionalidade através de vídeo. Como informações acionáveis coletadas a partir da análise de redes e grafos, lista: "Identify key opinion leaders; see relationship \& communities that matter; improve communication plan".

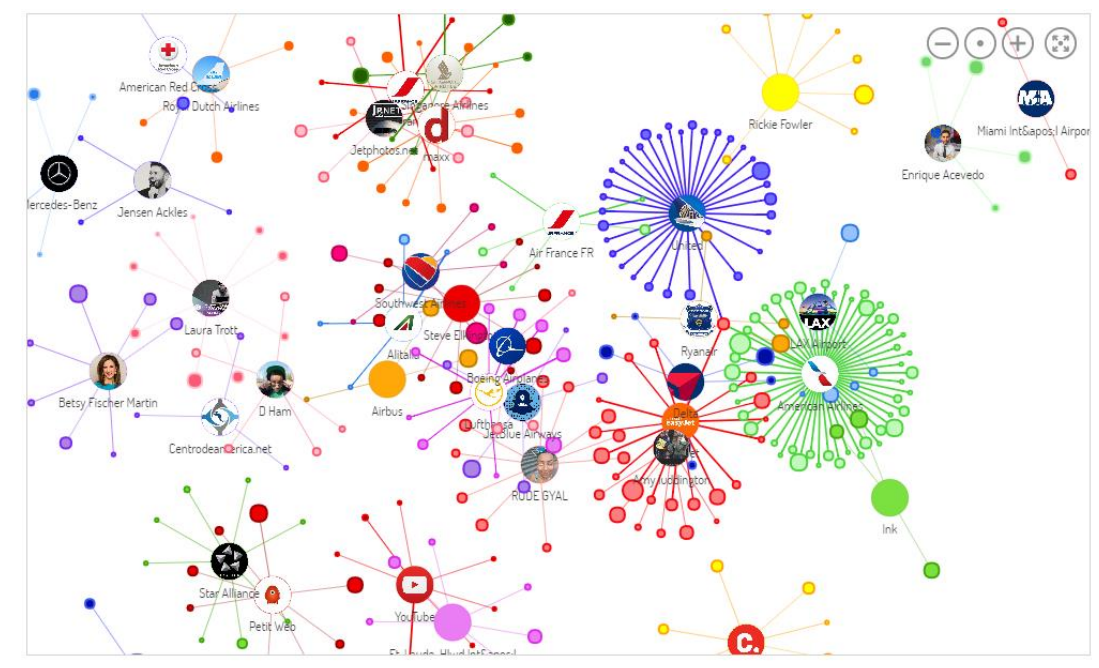

Figura 13: Tela da ferramenta Digimind

Desse modo, empresas e suas agências de comunicação podem ser persuadidos pela inovação incremental contínua através de funcionalidades dos softwares. A apresentação desta funcionalidade é valorizada pela Goldbach Interactive ao listar as três tendências no segmento: monitoramento em tempo real; dashboards e insights públicos; e análise de redes. Quanto à última diz que "as empresas tentam compreender a dinâmica das redes sociais e até mesmo a capacidade de influência na audiência. Encontramos até análise de redes sofisticadas em ferramentas como Digimind e BrandCare. Estas últimas tornam possível a análise de vários tipos de redes (páginas do Facebook, Instagram e Twitter) possível."

Uma particularidade dos softwares as a service para uso de outras empresas em suas atividades-fim como monitoramento de mídias sociais, neste caso, é um caráter duplo da abordagem da inovação. Segundo a classificação do Manual de Oslo, existem quatro tipos de áreas de inovações nas empresas: produto, processo, marketing e organização (OECD, 1997). Como vimos nas telas acima, as funcionalidades de visualização de redes e grafos tratam-se de inovações incrementais de produto, trazendo uma diferenciação aos softwares que a apresentam, expandindo as possibilidades de análise de dados. Ao mesmo tempo, para o

${ }^{15} \mathrm{http}: / /$ www.digimind.com/news/digimind-launches-the-influencer-network-for-twitter/ 
cliente (consultorias, agências de publicidade e afins) que utiliza o software para realizar outros produtos para o cliente final (empresa comercial, instituição pública etc). Em alguns casos, esta inovação pode ser vista como de processo, como no argumento sobre a “descoberta de influenciadores” facilitada pela análise de redes.

Assim, a noção de beta perpétuo como traço particular da web 2.0 (O’REILLY, 2005) permeia os negócios criados em torno desse novo panorama. Na descrição do lançamento, a Pulsar fala da aplicação dos dados, que teriam como objetivo "to help the researcher make sense of information flows and audience behaviour before they go onto working on a full network analysis of the dataset.". Em outro texto intitulado "Funcionalidades que o V-tracker tem e sua ferramenta provavelmente não tem"16, a V-Tracker cita a funcionalidade com o argumento "Ideal para você que está cansado daquele mesmo relatório de positivo e negativo, os grafos dão diversos insights como integração e interação entre assuntos, grupos e outros".

Em todos os casos, a funcionalidade não apresenta um adicional nos planos oferecidos, tática comum no segmento como concordam Chukarova \& Mikhramova: "To succeed in the emerging market, service vendors will have to make the transition as painless as possible for the users, or even consider offering services for free" (2010, p.70)

\section{CONCLUSÕES}

O conceito de inovação incremental é peça chave no panorama do modelo de negócio de software as a service. As ferramentas de monitoramento de mídias sociais, que seguem o modelo SaaS e oferecem seus serviços a centenas de empresas e agências dividem um ambiente de alta competição. Mesmo com características em comum, buscam oferecer diferenciais marginais para fortalecimento de posicionamento no mercado.

As possibilidades e constrições midiáticas dos usuários estão inscritas em contexto mercadológico que se adaptou aos novos desenhos da comunicação digital, ao mesmo tempo que os influencia. Entender desenhos mercadológicos de ambientes interacionais (as mídias sociais, neste caso) para além da relação direta entre cidadão-usuário e plataforma comunicacional permite evoluir a compreensão das questões sobre instâncias comerciais da cibercultura.

\footnotetext{
${ }^{16} \mathrm{http}: / /$ www.vtracker.com.br/blog/2015/08/funcionalidades-que-o-v-tracker-tem-e-sua-ferramentaprovavelmente-nao-tem/
} 
Descrevemos e observamos aqui, como parte de projeto maior de pesquisa, a apresentação comercial de nova funcionalidade de análise e visualização de redes e grafos sociais, apontada como tendência em rankings e avaliações de segmento da tecnologia. Ao estudar os modos pelos quais os fornecedores descrevem os lançamentos da inovação, o caráter de aplicação direta e otimização do trabalho são enfatizados e dividem espaço com referências à metodologia e software livre que os inspiraram.

\section{REFERÊNCIAS}

AMPOFO, Lawrence. The Social life of real-time social media monitoring. Participations, v. 8, n. 1, p. 21-47, 2011.

AMPOFO, Lawrence et al. HALFPENNY, Peter; PROCTER, Rob (Ed.). Innovations in digital research methods. Sage, 2015.

BACKSTROM, Lars; BOLDI, Paolo; ROSA, Marco; UGANDER, Johan; VIGNA, Sebastiano. Four Degrees of Separation. Proceedings of the 4th Annual ACM Web Science Conference. ACM, 2012. p. 33-42.

BATRINCA, Bogdan; TRELEAVEN, Philip C. Social media analytics: a survey of techniques, tools and platforms. AI \& SOCIETY, v. 30, n. 1, p. 89-116, 2015.

BEER, David. Using social media data aggregators to do social research. Sociological Research Online, v. 17, n. 3, p. 10, 2012.

BOYD, D. M;, ELLISON, N. B. Social network sites: Definition, history, and scholarship. Journal of Computer-Mediated Communication, 13(1), article 11, 2007.

BOYD, Danah. Social Network Sites as Networked Publics: Affordances, Dynamics, and Implications. In: PAPACHARISSI, Zizi (Ed.). A networked self: Identity, community, and culture on social network sites. Routledge, 2010.

BRUNS, Axel. How long is a tweet? Mapping dynamic conversation networks on Twitter using Gawk and Gephi. Information, Communication \& Society, v. 15, n. 9, p. 1323-1351, 2012.

CHUKAROVA, Inna; MIKHRAMOVA, Ramilka. Software as a Service: Study and Analysis of Saas Business Model and Innovation Ecosystems. Dissertação de Mestrado da Faculdade de Economia da Universiteit Gent, 2010.

EDUNOV, Sergey; DIUK, Carlos; FILIZ, Ismail; BURKE, Moira. Three and a half degrees of separation. Research at Facebook. Online, 2016. Disponível em: https://research.facebook.com/blog/three-and-a-half-degrees-of-separation/

ELLISON, Nicole; BOYD, Danah. "Sociality through Social Network Sites." In The Oxford Handbook of Internet Studies (Ed. William H. Dutton). Oxford: Oxford University Press, 2013.

GILliATT, Nathan. Social Media Analysis. Website. Acesso em julho de 2016. Disponível em: http://socialmediaanalysis.com/directory/ 
GRUBLBAUER, Johanna; HARIC, Peter. Social media monitoring tools as instruments of strategic issues management. In: FRIEDRICHSEN, Mike; MÜHL-BENNINGHAUS, Wolfgang. Handbook of social media management. Heidelberg: Springer Science+ Business Media, 2013.

KIETZMANN, Jan; CANHOTO, Ana. Bittersweet! Understanding and managing electronic word of mouth. Journal of Public Affairs, v. 13, n. 2, p. 146-159, 2013.

LAINE, Mikko OJ; FRÜHWIRTH, Christian. Monitoring social media: tools, characteristics and implications. In: International Conference of Software Business. Springer Berlin Heidelberg, 2010. p. 193-198.

LEMOS, André. Cibercultura. Alguns pontos para entender nossa época. In: LEMOS, André; CUNHA, Paulo (orgs). Olhares sobre a Cibercultura. Porto Alegre: Sulina, 2003; p. 11-23.

LEMOS, André. Cibercultura como território recombinante. A cibercultura e seu espelho: campo de conhecimento emergente e nova vivência humana na era da imersão interativa. São Paulo: ABCiber, p. 38-46, 2009.

NETSCIED. Network Literacy: Essential Concepts and Core Ideias. 2015. Disponivel em https://sites.google.com/a/binghamton.edu/netscied/teaching-learning/network-concepts

OECD. Manual de Oslo: Diretrizes para a Coleta e Interpretação de Dados sobre a Inovação. 1997. Disponível em http://www.finep.gov.br/images/apoio-e-financiamento/manualoslo.pdf

O'REILLY, Tim. O que é Web 2.0: padrões de design e modelos de negócios para a nova geração de software. Disponível in http://www. cipedya. com/doc/102010. 2005.

POLLOCK, Neil; D'ADDERIO, Luciana. Give me a two-by-two matrix and I will create the market: Rankings, graphic visualisations and sociomateriality.Accounting, Organizations and Society, v. 37, n. 8, p. 565-586, 2012.

RECUERO, Raquel; ZAGO, Gabriela; BASTOS, Marco Toledo. The discourse of\# ProtestosBR: content analysis of Twitter. Galáxia (São Paulo), v. 14, n. 28, p. 199-216, 2014.

REGATTIERI, Lorena et al. As Formas Perspectivas no Twitter: uma técnica quanti-qualitativa para estudos de Redes Sociais. Anais do XXXVII Congresso Brasileiro de Ciências da Comunicação - Foz do Iguaçu - 2 a 5/9/2014.

SILVA, M. F; JUNQUEIRA, L.A.P.; CARDOSO, O. Inovação e a Teoria Institucional. Revista Gestão.Org - Revista Eletrônica de Gestão Organizacional, v. 14, n. 1, 2016, p. 106-114.

SIRI, Júnior. O profissional de inteligência de mídias sociais no mercado brasileiro. 2015. Disponível em http://pt.slideshare.net/juniorsiri/profissional-de-inteligencia-de-mdias-sociais

STAVRAKANTONAKIS, Ioannis et al. An approach for evaluation of social media monitoring tools. Common Value Management, v. 52, n. 1, p. 52-64, 2012. 
Original recebido em: 19 de dezembro de 2016

Aceito para publicação em: 08 de abril de 2017

\section{Tarcízio Roberto da Silva}

Doutorando pela UMESP (com apoio taxa CAPES/PROSUP) e mestre em Comunicação e Cultura Contemporâneas pelo PPGCCC-UFBa. Cofundador e Diretor de Pesquisa em Comunicação do Instituto Brasileiro de Pesquisa e Análise de Dados. Professor convidado de MBAs e especializações (como Digicorp-USP, Unisinos e FBB). Co-organizador de livros como Para

Entender o Monitoramento de Mídias Sociais (Bookess, 2012) e Monitoramento e Pesquisa em Mídias Sociais: metodologias, aplicações e inovações (Uva Limão, 2016). Experiência em agências digitais, ferramentas de monitoramento e pesquisa aplicada de inovação (Social Figures, Flagcx, Coworkers, PaperCliQ e outras).

Esta obra está licenciada sob uma Licença Creative Commons. 